\title{
A Parallel Inference Method of Fuzzy Rules using Transputers
}

\author{
Non-member Sang Gu Lee (Hannam University ) \\ Member Kageo Akizuki (Waseda University )
}

\begin{abstract}
During the past decade, several specific hardware systems for fast fuzzy inference have been developed. However, most of them are dedicated to be executed sequentially, rule by rule, although they have parallel processing manners in operations of AND/OR computations. In this paper, we propose a parallel inference method of processing several fuzzy rules simultaneously by using T800 Transputer system, and analyze the worst case upper bound for internode message delays. The proposed control system has a relatively higher speedup, compared with the results of experiments in a single processor. This parallel inferencing fuzzy system can be used in a system requiring a rapid inference time in real-time system and/or one that has many inference variables in the condition part and the consequent part. Also, this system will be applied to build powerful architectures for control applications like robot control with time-critical sensor integration.
\end{abstract}

\section{Keywords: parallel fuzzy inference, Transputer, pendulum-car system}

\section{Introduction}

Since Zadeh introduced the fuzzy set theory in 1965, this theory has being applied to many areas. Fuzzy control has emerged as one of the most active and fruitful areas for research in the application of fuzzy set theory. Fuzzy logic, which is the logic on which fuzzy control is based, is much closer in spirit to human thinking and natural language than the traditional logical systems. Also, it provides an effective means of capturing the approximate and inexact nature of the real world [8]. Fuzzy logic control (FLC) is especially widely used in modern industry and many success stories have been reported $[6,8,9]$. Although achieving many practical successes, fuzzy control has not been viewed as rigorous science due to a lack of formal synthesis techniques which guarantee the very basic requirements of global stability and acceptable performance [14].

For FLC, the performance of controller is dependent upon the method of fuzzification, inference and defuzzification. Especially, inference method affects the performance of controller greatly, basically requires parallel characteristics and is possible in parallel processing. During the past decade, several specific hardware systems for fast fuzzy inference have been developed. However, most of them are dedicated to be executed sequentially, rule by rule, although they have parallel processing manners in operations of AND/OR computations. Therefore, it is necessary to parallelize fuzzy rules themselves.
This paper shows a parallelizing method of the inference part for the rules of fuzzy control, a core of fuzzy controls, through T800 transputers system. For transputers, there are two kinds of linking methods, static and flood-fill $[4,10]$. Regarding each of these methods, realization of parallel fuzzy control systems with these two linking methods are presented, and applied to the stabilization of control system of an pendulum-car problem. To evaluate and compare performances of the proposed parallel fuzzy control system, fuzzy control system for single processor performing the same function is implemented and compared to multi-transputer system. Simulation results are obtained for each implemented system.

Section 2 briefly reviews T800 transputer, and Section 3 describes the parallel inference method of fuzzy rules. Section 4 presents an application example and experimental result of pendulum-car system, and Section 5 is conclusion.

\section{T800 Transputer}

T800 Transputer (TRANSister comPUTER) is a multi-computer building block developed by INMOS and it is the microcomputer designed specifically for use in parallel processing systems. The organized network is inter-connected to one host computer. Transputer system is an MIMD machine in that programming is independently possible for each processor. There are $4 \mathrm{~KB}$ fast on-chip static RAM, a 32-bit integer CPU and a 32-bit IEEE FPU (floating 
point unit) in T800 transputer. CPU and FPU are operated in parallel. In T800 transputer, there are 4 link interfaces, where links are DMA-controlled, bidirectional, serial transmission channels, and up to 9MB I/O throughput is supported per each transputer [4]. T800 transputer can be configured in a variety of topologies with less than four fan-in/fan-out such as a four dimensional $\mathrm{N}$-cube, a binary or ternary tree, a flat grid or torus. A transputer can also process multiple threads through time slicing.

There are two linking methods for T800 transputers [4]. Firstly, static method is what, in advance on the configuration file, a user explicitly set up the relationship between the processors on which the task is undertaken and the task to be done on the transputer network. The method has an advantage that a programmer can organize a structure of network in a topology as he wants, and has a disadvantage that he must supervise a great part of network personally. Secondly, flood-fill method, where an application is divided into a master and a worker task, loaded automatically into a certain transputer network by afserver program. In this method, when a transputer is added to the network, recompilation and reconfiguration is not necessary, the processing speed will thus be faster, and acceptable to applications which require a burdensome calculation.

\section{Parallel inference method of fuzzy rules}

The most important advantages of FLC are: intuitive linguistic design, reflecting the behaviors of expert, the fact that the model of the controlled process is not necessary, and good quality control. However, the main disadvantages of FLC are: the need for expert's knowledge about the controlled object, sequential process through rule bases, and time-consuming defuzzification process. FLC is based on the rules which express expert's knowledge about the dynamics of control system as linguistic forms. In the case of having many input and output variables, it is good to parallelize each variable and to inference many active rules simultaneously.

Generally, the rule of fuzzy control takes a form of IF (set of conditions are satisfied) THEN (set of consequences can be inferred).

In the case of fuzzy control system with two inputs and one output, control rules are

$$
\begin{gathered}
R_{1}: \text { if } x \text { is } A_{1} \text { and } y \text { is } B_{1} \text { then } z \text { is } C_{1} \\
R_{2}: \text { if } x \text { is } A_{2} \text { and } y \text { is } B_{2} \text { then } z \text { is } C_{2} \\
\ldots \ldots \ldots \ldots \ldots \ldots \\
R_{n}: \text { if } x \text { is } A_{n} \text { and } y \text { is } B_{n} \text { then } z \text { is } C_{n} .
\end{gathered}
$$

Here $x, y$ and $z$ are linguistic variables representing two input variables and one control variable, and $\boldsymbol{A}_{i}, \boldsymbol{B}_{\boldsymbol{i}}$ and
$C_{i}$ are linguistic values of linguistic variables $x, y$ and $z$ on the universe of discourse $\boldsymbol{U}, \boldsymbol{V}$ and $\boldsymbol{W}$ where $i=1$, $2, \ldots, n$.

If the number of input variables is $\boldsymbol{n}$ and there are $\boldsymbol{m}$ linguistic values for each variable, then the number of maximum possible control rules will be $m^{n}$. The overlap factor $\boldsymbol{d}$ is the maximum number of an input variable's linguistic values that overlap in their membership functions [3]. In many cases, $d$ is 2 for most linguistic variables in most applications. The maximum number of active rules that can be operated simultaneously is typically smaller than $d^{n}$. For example, with 4 input variables and an overlap factor of 2 for each variable, the possible number of concurrent active rules is $2^{4}=16$.
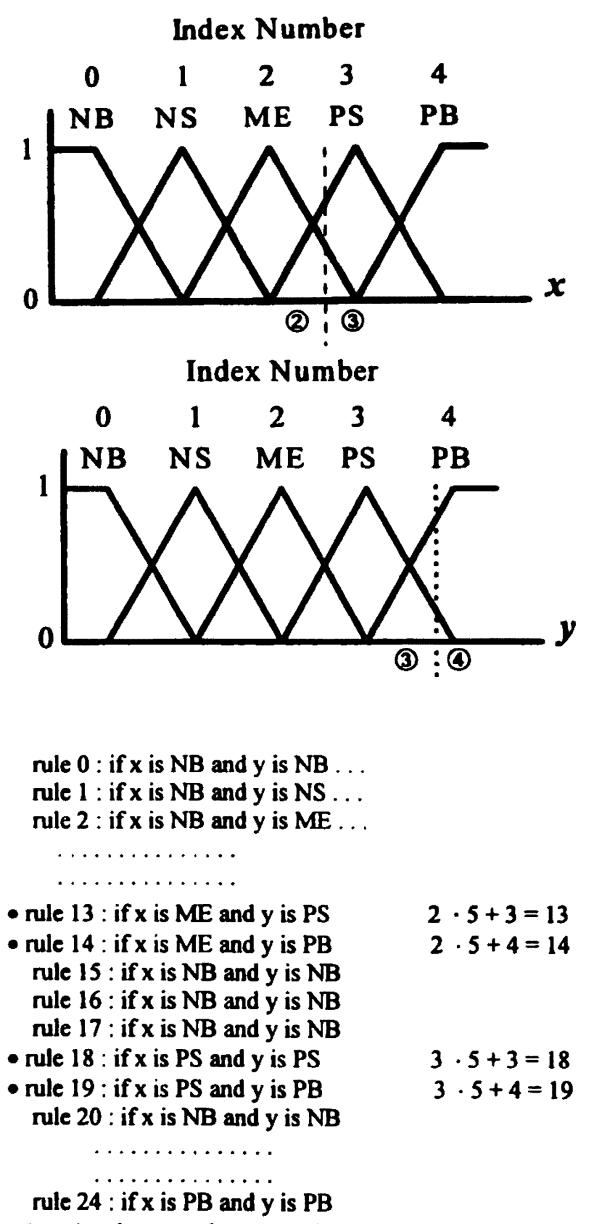

Fig.1 Active rule numbers that can be processed simultaneously

The method of determining the rule numbers of concurrent active rules is as follows. For example, we consider the case of 2 input variables and 5 linguistic values per each variable, and sensed input values are shown in Fig. 1. The index numbers are $0,1,2,3$ and 4 
corresponding to NB, NS, ME, PS and PB, respectively. In the first input variable $x$, linguistic values of $\mathrm{ME}(2)$ and $\mathrm{PS}(3)$ are active. In the second input variable $y$, linguistic values of $\mathrm{PS}(3)$ and $\mathrm{PB}(4)$ are active. Therefore, only 4 rules are necessary among the 25 rules in fuzzy inference process at each sampling time. Pairs of active rules are $(2,3),(2,4)$, $(3,3)$ and $(3,4)$. If a pair is $(p, q)$, the corresponding rule number is computed as

$$
p \times \text { (number of linguistic values) }+q \text {. }
$$

Therefore, rule numbers $13,14,18$ and 19 can be processed simultaneously. The rules except above 4 rules are no more needed at the processing. In the proposed method, there is no need to search all the fuzzy rules.

Taking these points into consideration, we can parallelize the computations of the fuzzy logic control system. As a necessary condition of parallelization, the optimum arrangement of membership functions of linguistic values for a linguistic variable maintaining completeness is as follows:

(1) The height of maximum overlap grade between two adjacent membership values is not limited to 0.5 .

(2) More than three linguistic values must not be overlapped.

(3) Each linguistic value has its own index number.

To make the fuzzy inferencing in parallel and easy for fuzzy control of the transputer system, the memory space of each processor should be allocated and initialized as Fig. 2. First, memories are allocated for each linguistic variables, and membership functions corresponding to the linguistic values of the variable are stored. The size of memories depends upon how many levels are quantized in the domain.

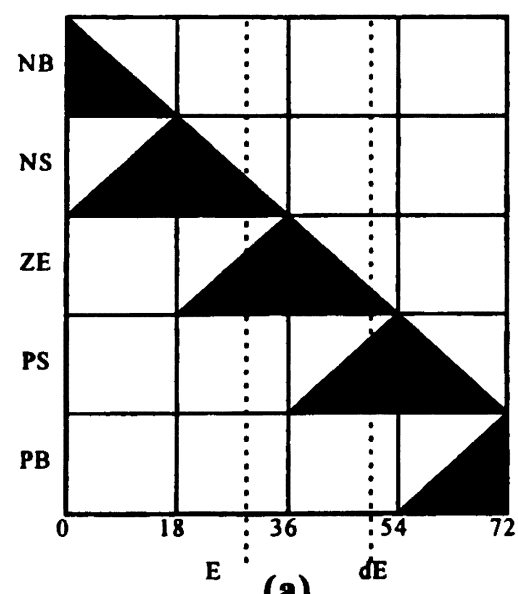

(a)

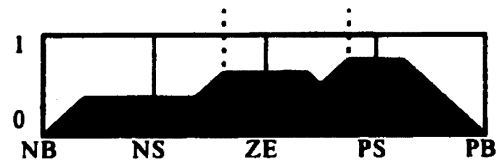

(b)

\begin{tabular}{|c|c|c|c|c|c|}
\hline $\mathrm{dE}$ & NB & NS & $Z E$ & PS & PB \\
\hline NB & NB & NB & NB & NS & ZE \\
\hline NS & NB & NB & & & PS \\
\hline ZE & NB & NS & & & PB \\
\hline PS & NS & ZE & PS & PB & PB \\
\hline PB & ZE & PS & PB & PB & PB \\
\hline
\end{tabular}

(C)

Fig. 2

(a) structure of linguistic variable,

(b) memory space for storing inference results, and

(c) control rule storage for the control rule

Second, control rules are to make an array where the number of input variable is in order and the number of linguistic values of corresponding variable is the number of elements, and to store linguistic values of the conclusion part. Finally, memory spaces for storing inference results are allocated, where the size of memory is the number of quantization for the domain.

There are two ways to achieve the realization of parallel fuzzy control systems according to the link structure of the transputers. These are the flood-fill and the static methods.

\subsection{Parallel fuay inference system by flood-fil method}

In flood-fill method, the application is divided into master and worker and loaded into a transputer network by afserver program. Master and worker tasks are processed according to the following algorithm.

<Master>

(1) input each variable's data from sensors.

(2) get quantized value by multiplying constant scaling factor by input value according to the domain set up by each variable.

(3) obtain linguistic value and grade that will be used for inference by quantized value

(4) determine control rules by combining the indices of linguistic values obtained from (3) and gets linguistic value of consequent part.

(5) request inference tasks of $\boldsymbol{d}^{n}$ to each worker.

(6) merge returned values from each worker.

(7) defuzzify and apply to system.

(8) go to (1)

\section{$<$ Worker $>$}

(1) receive linguistic value and grade of the consequent part from master.

(2) generate the results for the linguistic value 
according to the grade.

(3) return the results.

The flowcharts of processing for the master, worker, send thread and receive thread are as following Fig. 3.

\subsection{Parallel fuezy inference system by static method}

Static method is a way of making parallel application for the transputers system with fixed hardware link configuration. According to the topology a user is to use, task and wiring that will be done on the transputer

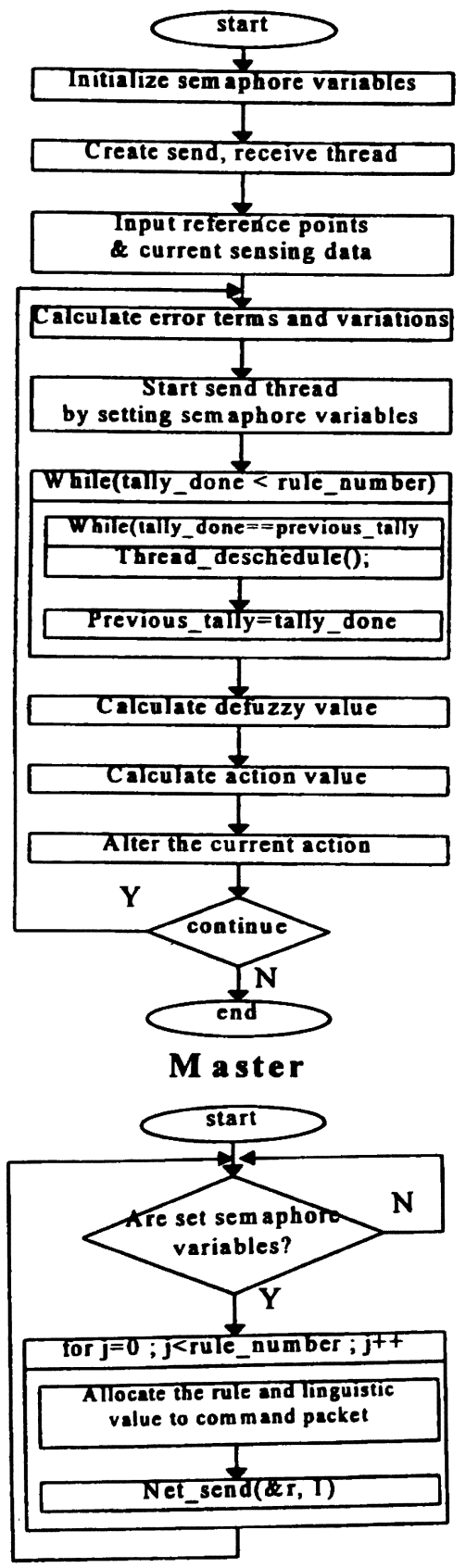

Send Thread
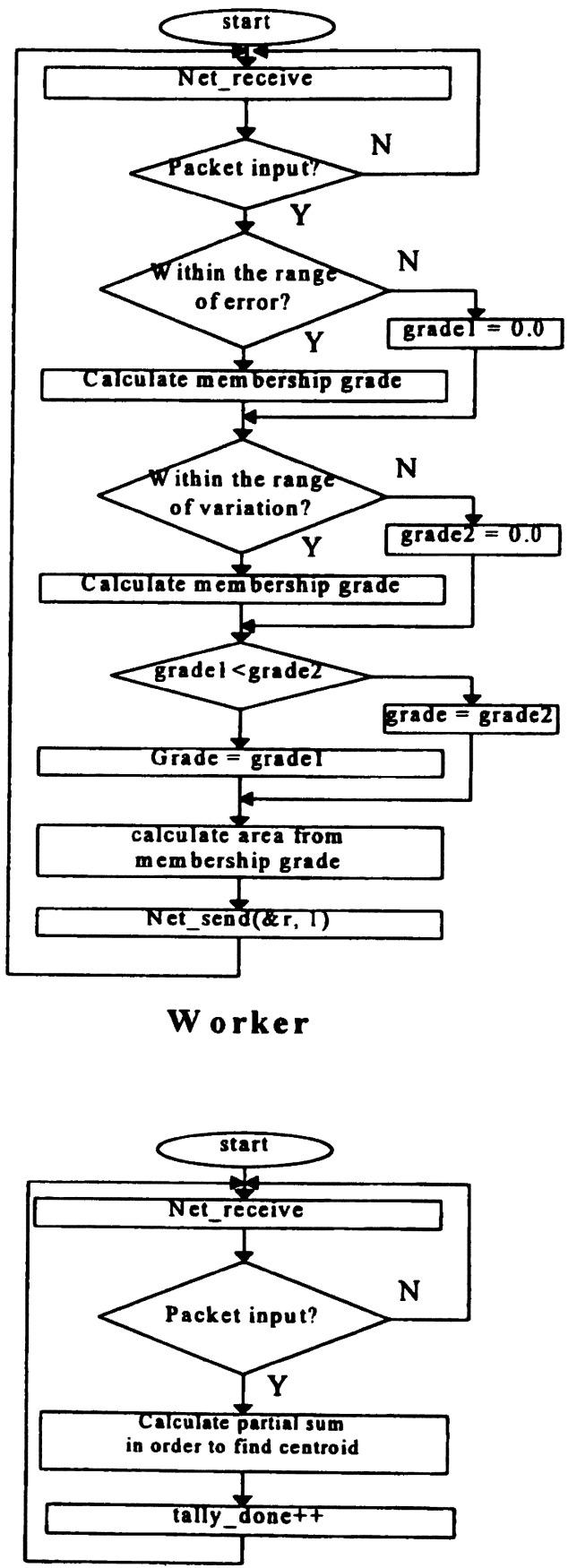

Receive Thread

Fig. 3 Parallel fuzzy inference for the flood-fill method network is set beforehand on the configuration file. In this experiment, up to 16 transputers were used. And one of the transputers is for root processor as a master and the others are thus used for parallel inference processing as workers. So, for this usage, wiring is set up. 


\section{Example and experimental result}

The proposed method is applied to the problem of stabilization of pendulum-car system that has been widely used as an integrated experimental tool dealing with application problem of modern control system $[1,2]$. Pendulum-car system and its model for the parallel fuzzy system are shown in Fig. 4.

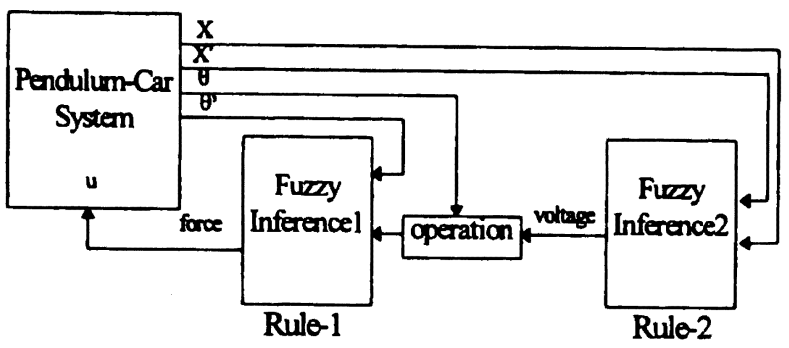

Fig. 4 Model of pendulum-car system

Here, Pendulum-car system is expressed by the following four state variables;

$$
\begin{gathered}
S=\left[X, X^{\prime}, \theta, \theta^{\prime}\right]^{T} \\
X: \text { car's position } \\
X^{\prime}: \text { car's velocity } \\
\theta: \text { pole's angular position } \\
\theta^{\prime}: \text { pole's angular velocity }
\end{gathered}
$$

By the above four state variables, control value $u$ is determined, and the goal of the system is to make $\theta=$ $0, X=0$. The relationship between state variable $S$ and control variable $u$ in time $t$ is represented as a function, $u(t)=F(S(t))$ and to make $\theta$ and $X$ approach 0 within a proper time by the control of $u(t)$. Here rule-1 balances the pendulum and rule- 2 positions the car to the center of its track. A controllable motor moves the car in either direction according to the control signal sent to it. The control signal is represented by the variable volt, the voltage of the signal sent to the motor. The car moves to the left if volt is positive.

For the fuzzy logic control system, the input variables $X(t), X(t), \theta(t), \theta(t)$ and output variable $u(t)$ of controller use seven linguistic values such as NL(Negative Large), NM(Negative Medium), NS(Negative Small), ZE(Zero), PS(Positive Small), PM(Positive Medium) and PL(Positive Large). The domain of all membership functions is normalized in 0 $\sim 255$ or $-127 \sim+127$. And the overlap factor of two adjacent linguistic values is 2 . We use the Mamdani's method for fuzzy inference and center of gravity as defuzzification for the generating the control action.

Using these concepts, two cases of parallel fuzzy control systems, such as flood-fill and static method, are realized in this paper by means of the methods presented above. And, fuzzy control system for single processor is compared to evaluate the performance of parallel fuzzy control system.

In each case of fuzzy control system, the initial parameter values were set as follows.

$$
\begin{aligned}
& X=1.5 \quad / * \text { car's position */ } \\
& \theta=3.5 \quad I^{*} \text { angular displacement from vertical */ } \\
& g=9.81 \quad / * \text { gravity constant }(\mathrm{m} \mathrm{/} \mathrm{s}) * / \\
& M=2.0 \quad / * \text { mass of the car }(\mathrm{kg}) * / \\
& m=0.1 \quad / * \text { mass of the pole }(\mathrm{kg}) * / \\
& L=1.0 \quad / * \text { length of pole }(\mathrm{m}) * 1 \\
& f=8.0 \quad I^{*} \text { fraction coefficient }(\mathrm{kg} / \mathrm{s}) * / \\
& u=0 \quad / * \text { external horizontal force applied on the car } * /
\end{aligned}
$$

In the experiment of this research, PARA16 system that is composed of 16 T800 transputer modules was used. In PARA16, transputers are connected to each other through C004 crossbar switch. In our experiment, transputer mesh structure was used to provide maximum utilization in links between processors. Before the execution of a parallel program, given fuzzy rules are distributed to n-node and allocated to each processor at the same size. The number of tasks statically allocated initially is the same. However, the computing time of each task becomes gradually different to each other and loads of each processor vary. In this paper, we do not consider the problem of the dynamic load balancing. In our experiment, we measured the times to reach the goal of the system and gained the results in the case of 4,8 and 16 processors, respectively. Fig. 5 shows speedup of the two methods, and the performance between transputers system in the flood-fill method and a general microprocessor is shown in Table 1.

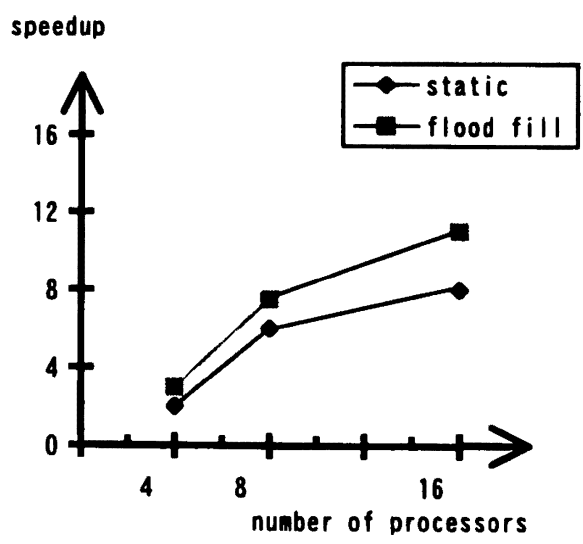

Fig. 5 Speedup

Comparing the architectures of today's special fuzzy processor and a general microprocessor shows that the latter such as the $80 \times 86$ or the $680 \mathrm{X0}$ are faster than the former [13]. Therefore, we did not compare the proposed method with the special fuzzy processor 
using fuzzy chips like Togai FC110.

Table 1. Performance

\begin{tabular}{|c|c|c|c|c|}
\hline \multirow{2}{*}{} & \multicolumn{3}{|c|}{ Transputers(25MHz) } & \multirow{2}{*}{ PC486 } \\
\cline { 2 - 4 } & 1 & 4 & 16 & $(150 \mathrm{MHz})$ \\
\hline Time(Sec.) & 67.32 & 23.54 & 6.21 & 81.37 \\
\hline Speedup & 1 & 2.86 & 10.84 & \\
\hline Efficiency & 1 & 0.72 & 0.68 & \\
\hline
\end{tabular}

Flood-fill method is faster than static method, and even more faster than general processor. Speedup factor is less than the number of processors. This is caused by the communication delay of tasks. In transferring of tasks, if the task size is large, then migration and overhead of routing become relatively large. Storing and transferring of large data at each node are the factor in degrading the performance in the case that router uses the store-and-forward method.

If the size of a task to be transferred is $x$ bytes, the cost of transferring a task from node $A$ to node $B$ is cost $=$ distance $(\mathrm{A}, \mathrm{B}) \cdot \boldsymbol{x} \cdot \boldsymbol{a}$

where distance $(A, B)$ is the distance between $A$ and $B$, and $a$ is routing cost factor.

To provide fast transfer times, tasks must be split into packages of known constant length. In general, the delay-time of a message with an arbitrary length of $k$ bytes is

$$
\begin{aligned}
\mathrm{T}_{\text {delay }}= & \mathrm{p} \cdot \mathrm{T}_{\text {setup }}+\mathrm{p} \cdot \sum_{\mathrm{i}=1}^{\mathrm{m}}\left(\mathrm{T}_{\text {routing }}(\mathrm{i}) \cdot \mathrm{s}+\mathrm{T}_{\mathrm{i}}\right) \\
& \mathrm{p}=\text { number of package } \\
& \mathrm{s}=\text { size of package } \\
& \mathrm{m}=\text { number of intermediate node } \\
& \mathrm{T}_{\mathrm{i}}=\text { runtime of routing process on node } \mathrm{i} \\
& \mathrm{T}_{\text {setup }}=\text { time needed for message startup }
\end{aligned}
$$

In PARA16, transputers system is composed of 16 T800-25 transputers with a linkspeed of 20Mbit/s. Topology of this system is $4 \times 4$ mesh. We tested the performance of communication system of PARA16. The delay time is measured between the leftmost node and the rightmost node in the second row.

The average delay time is measured for one package. Considering equation (1), having a startup-time $T_{\text {setup }}$ of about $150 \mu \mathrm{s}$ and an internode latency of $120 \mu \mathrm{s}$, we expect a message delay time of

$$
\text { Tmessge }=150 \mu \mathrm{s}+\sum_{\mathrm{j}=1}^{2} 120 \mu \mathrm{s}=390 \mu \mathrm{s}
$$

And the worst case is an additional delay-time of another two internode latencies on the second node. Thus, we can predict an upper bound for the delay time.

$$
\mathrm{T}_{\text {worst_case }}=150 \mu \mathrm{s}+\sum_{\mathrm{j}=1}^{2+2} 120 \mu \mathrm{s}=630 \mu \mathrm{s}
$$

According to our performance tests, the parallel fuzzy inference system provides fast data transfer and operation. Even if the network is heavily loaded, we can predict an upper bound for the message delay, which is important in hard real-time applications. Minimizing the runtime of the routing processes and using packages of constant length drastically decrease the internode latency to $120 \mu \mathrm{s}$.

\section{Conclusion}

In this paper, we have developed a parallel inference method of fuzzy rules using T800 Transputer system for the fast fuzzy inference system. We also analyzed the worst case upper bound for the internode message delay. The proposed system has the following features:

- Several fuzzy rules are executed simultaneously.

- This system brought a relatively higher speedup, compared with the results of experiments come from adapting into the single processor.

- In parallel fuzzy inference methods in this paper, the flood-fill method is especially thought to be more acceptable to a system which requires short sampling time and fast response.

This parallel fuzzy inference system can be used for a system requiring a rapid inference time in real-time system and/or has many inference variables in condition part and consequent part. This system will be applied to build powerful architectures for control applications like robot control with time-critical sensor integration.

(Manuscript received Oct. 23, '97, revised Feb. 13, '98)

\section{References}

[1] B. Bavarian, B. F. Wyman and H. Hemami, "Control of the Constrained Planar Simple Inverted Pendulum," Int. J. Control, Vol. 37, No. 4, pp. 741-753, 1983.

[2] N. S. Bedrossian, "Approximate Feed-Back Linearization : The Cart-Pole Example," Proc. of the 1992. IEEE Int. Conf. on Robotics and Automation, pp. 1987-1992, 1992.

[3] T-C Chiueh, "Optimization of Fuzzy Logic Inference Architecture," Computer, pp. 67-70, May, 1992.

[4] R. S. Cok, Parallel Programs for the Transputer, Eastman Kodak Company, 1991.

[5] O. Ishizuka, H. Tanno, Z. Tang, H. Matsumoto, "Optimum Control Based of Fuzzy Inference and its Simulation," Proc. of the Int. Conf. on 
Fuzzy Logic \& Neural Networks, pp.61-64, July, 1990.

[6] A. Kandel, L. Z. Cao, "Fuzzy Inference and its Applicability to Control Systems," Fuzzy Sets and Systems, Vol. 48, pp. 99-111, 1992.

[7] S-G Lee and K. Akizuki, "Design of an Effective Parallel Architecture for Fuzzy Information Processing," Proc. of IASTED Int. Conf. Artificial Intelligence and Soft Computing, pp. 292-295, Jul. 1997.

[8] C. C. Lee, "Fuzzy logic Control Systems: Fuzzy Logic Controller-part I, II," IEEE Trans. Syst. Man Cybern., Vol.20, No.2, pp. 404-435, 1990.

[9] E. H. Mamdani, "Application of Fuzzy logic to Approximate Reasoning Using Linguistic Synthesis," IEEE Computer, Vol C-26, No.12, Dec. 1992.

[10] J. M. J. Murre, "Transputers and Neural Networks: An Analysis of Implementation Constraints and Performance," IEEE Trans. on Neural Networks, vol.4, No.2, pp.284-292, 1993.

[11] N. Pfluger, J. Yen and R. Langari, "A Defuzzification Strategy for a Fuzzy Logic Controller Employing Prohibitive Information in Command Formulation," IEEE Int. Conf. on Fuzzy Systems 1992.

[12] H. Surmann et al., "What kind of Hardware is Necessary for a Fuzzy Rule Based System," Proc. FUZZ-IEEE World Congress Computational Intelligence, Vol. 26, No.6, pp.274-278, 1994.

[13] H. Surmann and A. P. Ungering, "Fuzzy RuleBased Systems on General Purpose Processors," IEEE Micro, pp.40-48, Aug. 1995.

[14] L. X. Wang, "Stable Adaptive Fuzzy Control of Nonlinear Systems," IEEE Trans. on Fuzzy Systems. Vol.1, No.2, pp. 146-154, 1993.

[15] T. Yamakawa, "A Fuzzy Inference Engine in Nonlinear Analog Mode and Its Application to a Fuzzy Logic Control," IEEE Trans. on Neural Networks Vol.4, No.3, pp. 496-522, 1993.

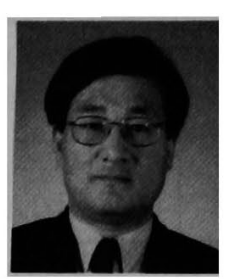

Sang Gu Lee (non member) is currently a professor of Hannan University, Korea, since 1983 . He is now engaged in research of parallel architecture for fuzzy and neuro processing. He is a member of the IEEE, and an international program committee of the Artificial Intelligence and soft Computing of IASTED.

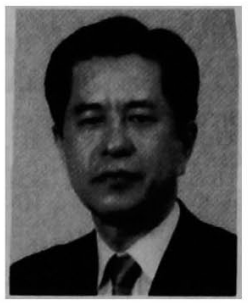

Kageo Akizuki (member) received the B.E., M.E. and Dr. of Engineering degrees all in Electrical Engineering from Waseda University, Tokyo, Japan, in 1958, 1960 and 1965, respectively. Since 1963 , he has been with Waseda University, where he is now engaged in research of system control theory and signal processing, and is a professor. He is a member of the IEEE, and the IEE of Japan. He is a chairman of the Electronics, Information and System society of the IEE of Japan. 